electrodynamics in vacuo; 7. waves; 8 . relativistic mechanics of continuous matter; appendix, tensors for special relativity. Some omitted topics: variational principles for Maxwell's equations and other field theories, and the relation between Lorentz invariance of the action and the existence of conservation laws; spinors and the structure of the Lorentz group. There is a good collection of interesting and stimulating exercises, many with hints and answers. There remains one puzzling question: Why should a book, priced at $10 \mathrm{~s} .6 \mathrm{~d}$ in Great Britain, be priced at $\$ 2.25$ in the U.S.?

\title{
Alfred Schild
}

Seminar on transformation groups. By A. Borel with contributions by G. Bredon, E. E. Floyd, D. Montgomery and R. Palais. Annals of Mathematics Studies no. 46. Princeton University Press, 1960. 245 pp. $\$ 4.50$.

A topological transformation group is a triple $(G, X, \pi)$ consisting of a topological group $G$, a space $X$, and a map $\pi: G \times X \rightarrow X$ such that $\pi(e, x)=x$ for all $x \in X$ and $e$ the identity of $G$ and such that $\pi\left(g_{1}, \pi\left(g_{2}, x\right)\right)=\pi\left(g_{1}, g_{2}, x\right)$ for all $g_{1}, g_{2}$ in $G$ and $x \in X$. It is customary to omit $\pi$ and write $(G, X)$ and $\pi(g, x)=g x$. We may regard $G$ as represented as a group of homeomorphisms of $X$ onto itself. In this manner we might regard $(G, X)$ as an extension of the concept of linear (matrix) representations. It is also convenient to regard transformation groups as a generalization of principal fibre bundles. This latter interpretation is suggested by the introduction of the orbit space $X \mid G$ and the quotient map $\nu: X \rightarrow X \mid G$.

In this Annals Studies the group $G$ is taken to be a compact Lie group (possibly finite) and various conditions are imposed on $X$. Two of the main questions that can be pursued for transformation groups concern the nature of the fixed point set; that is, the set of points in $X$ such that $g x=x$ for all $g \in G$, and the structure of the orbit space, $X \mid G$. There are, of course, many other interesting questions, and we have mentioned these two only to give us a start.

The first two chapters deal with generalized manifolds and, in themselves, have no relation to transformation groups. The basic idea, exploited so extensively by Wilder, is to extract certain local and global properties from locally euclidean spaces which can be expressed purely in terms of algebraic topology and to show that when these properties are imposed on an abstract space then the space, again from the point of view of algebraic topology, will exhibit the characteristics of a locally euclidean space. The idea is to impose "local" conditions and then to derive "global" results; for example, 
the Poincaré Duality Theorem. The first chapter sets up the definitions and the second handles the duality theorem. A noteworthy feature of Chapter 2 is the discussion of homology groups for locally compact spaces. This makes it possible to formulate a Poincaré duality theorem for generalized manifolds in which the coefficient ring is a principal ideal domain, but not necessarily a field. We shall briefly comment on the role played by generalized manifolds in the study of transformation groups.

If attention is focused on $G=Z_{p}$, the cyclic group of prime order, then we enter the P. A. Smith theory of periodic maps of prime order. In the third chapter, by Floyd, this Smith theory is discussed. The special cohomology groups are used to derive several well-known results. For example, let $X$ be a locally compact Hausdorff space for which $\operatorname{dim}_{Z_{p}} X<\infty$ and $H_{\phi}^{*}\left(X ; Z_{p}\right) \simeq H^{*}\left(S^{n} ; Z_{p}\right)$, where $\phi$ is a paracompactifying family. If $Z_{p}$ acts on $X$ and $F \subset X$ is the fixed point set, then there is an integer $-1 \leqq r \leqq n$ such that $H_{\phi}^{*}\left(F ; Z_{p}\right)$ $\simeq H^{*}\left(S^{r} ; Z_{p}\right)$. The presentation differs from that of Smith in that the special cohomology groups are derived from the Leray spectral sequence of a map.

Chapters 4 and 5, by Borel, are respectively titled The action of $Z_{p}$ or $T^{1}$ : Global theorems, and The action of $Z_{p}$ or $T^{1}$ : Local theorems. Here $T^{1}$ is the circle group. In the first of these chapters Borel presents his alternative approach to Smith theory which achieves the ends by considering the spectral sequence associated with a regular covering. It may be stated that as far as the action of $Z_{p}$ is concerned we can come to about the same conclusions by following either Chapter 3 or Chapter 4. The reviewer, however, knows no satisfactory explanation of this fact. Certainly the approaches do not appear to have much in common. For the action of the circle group $T^{1}$ the advantage is with Borel's techniques.

Chapter 5 discusses local cohomology properties of the fixed point set of an action of $Z_{p}$ which can be inherited from the space $X$. It is in this section that the connection between generalized manifolds and the action of $Z_{p}$ appears. The following, due to P. A. Smith, is shown. Let $X$ be a cohomology $n$-manifold over the field $Z_{p}$, and let $G$ be a finite $p$-group acting on $X$, then the fixed point set $F$ is also a cohomology manifold over $Z_{p}$, and is orientable if $X$ is. Now this illustrates the value in using cohomology manifolds because this property is inherited by the fixed point set of $Z_{p}$. If $X$ is locally euclidean, however, this need not be true of the fixed point set. Actually a little reflection shows that it is sufficient to show the quoted result for $Z_{p}$. Another point should be made also. The argument 
showing $F$ is a cohomology manifold involves a localization of the argument which shows that if $Z_{p}$ acts on a $\bmod p$ cohomology sphere then the fixed point set is also a $\bmod p$ cohomology sphere. Thus we see a global theorem about the fixed point set of an action of $Z_{p}$, together with its appropriate local version.

In Chapter 6 Floyd presents his extremely important result which in this form asserts that if a toral group $T^{k}$ acts on an $n$-cohomology manifold $X$ over $Z$ and if $C$ is a compact invariant subset of $X$, then $T^{k}$ has only a finite number of distinct isotropy groups in $C$. For a transformation group $(G, X)$ the isotropy subgroup at $x \in X$ is the subgroup of all $g \in G$ for which $g x=x$. There is no particularly simple description of the argument which involves regular convergence and the full impact of Smith theory. Up to this point we have not mentioned the action of toral groups, although such actions were discussed in Chapters 4 and 5. The more discerning of those results have as part of the hypothesis the conclusion of the Floyd theorem. Thus we have a large collection of spaces to which such results apply automatically. For example, if the circle group $T^{1}$ acts on a compact connected manifold $X$ the finiteness of the number of isotropy groups makes it possible to show that the orbit space $X \mid T$ is a compact ANR.

With Chapter 7, Finiteness of number of orbit types, by Bredon, we leave the Smith theory and go to general Lie groups acting. This chapter is a short exposition of Mostow's theorem that if a compact Lie group $G$ acts on a manifold then in any compact invariant subset of the manifold there are only a finite number of distinct orbit types. Chapter 8 , by Palais, is Slices and equivariant imbeddings. The reviewer here was surprised to find no reference to the work of Montgomery and Yang on the existence of slices. Actually the imbedding theorem of Mostow discussed in this chapter is a substantial justification of that point of view which regards transformation groups as a generalization of linear representations. A special case of the imbedding theorem is the following. Let $(G, M)$ denote a compact Lie group acting on a compact manifold. There is a linear representation of $G,\left(G, E^{n}\right)$ (on a sufficiently high dimensional Euclidean space) such that $(G, M)$ can be homeomorphically imbedded in $\left(G, E^{n}\right)$ by an equivariant homeomorphism.

The next chapter, 9, by Montgomery on Orbits of highest dimension, is a bit difficult to describe in a few sentences. It is an exposition of certain results of Montgomery, Samelson, Yang and Zippin concerning the highest dimensional orbits which appear in an action of a compact Lie group on a manifold. Recent work of Montgomery and Yang has built considerably on the material in this section. Probably 
this chapter could (and should) be read separately as background material for studying this Montgomery-Yang work.

Chapters 10 and 11 by Borel discuss spectral sequences and in particular the Fary sequence. These chapters are not directly concerned with transformation groups.

In Chapter 12, Borel discusses some fixed point theorems for elementary commutative groups. In a sense this returns us to Smith theory. Of course this is to be taken very loosely, since the specific results are due to Borel and really follow from what was earlier his general approach to Smith theory. An elementary abelian $p$-group ( $p$-prime) is a finite direct sum of the cyclic group $Z_{p}$ with itself. The number of factors is the $p$-rank. A number of interesting facts about the action of such groups are demonstrated. For one example, let $G$ be an elementary abelian $p$-group $(p \neq 2)$ acting on quaternionic projective space of real dimension $4 s$. Let $F$ be the fixed point set of this action, then $\sum_{0}^{4 s} \operatorname{dim} H^{i}\left(F ; Z_{p}\right)=S+1$. If there were only a finite number of fixed points, then there would be exactly $S+1$. In general a number of results about the fixed point set of actions of these elementary groups on projective spaces are given. Chapter 13 continues the action of elementary abelian groups, including some local results.

Chapter 14, by Borel, on One or two classes of orbits is worth some comment. Actually it shows up the serious problems which arise in considering the action of a general compact Lie group. For cyclic groups of prime order, $p$-groups, or elementary abelian groups it is possible to put some reasonable hypothesis on the space on which the group acts and to derive some conclusion about the fixed point set or the orbit space. Now in this chapter we see strong conditions placed on the space $X$ but in addition a very strong hypothesis is put on the way in which the Lie group acts. For one orbit type it is assumed that all isotropy groups are conjugate, while for two orbit types there are exactly two conjugacy classes of isotropy groups! This is not a negation of the results but merely an illustration of the problems arising in discussing the action of general groups. For example it is shown that if a compact Lie group acts on Euclidean space with exactly two orbit types, then $G$ actually has a fixed point. It is known by example, on the other hand, that even a finite group can act on Euclidean space without a fixed point.

Chapter 15, by Bredon on Fixed point sets of high dimension, is, in part, background material for some of his later work. This is the presentation of some original results by Bredon. He considers a compact connected Lie group $G$ acting on an $n$-manifold $M^{n}$ with highest 
dimensional orbit of dimension $k$. He assumes that the dimension of the fixed point set $F$ is exactly $n-k-1$, and looks at what happens. For example, if $M^{n}=S^{n}$, the $n$-sphere, then $F$ is an $(n-k-1)$ cohomology manifold over the integers with the integral cohomology of $S^{n-k-1}$. Furthermore the orbit space $S^{n} \mid G$ is a cohomology $(n-k)$ cell.

The final chapter, 16, by Borel, discusses the spectral sequence of a map. The reviewer concludes by expressing his hope that this book will stimulate more interest and activity in transformation groups.

P. E. CONNER

Integral functions. By M. L. Cartwright. (Cambridge Tracts in Mathematics and Mathematical Physics, no. 44). Cambridge, at the University Press, $1956.8+135$ pp. $\$ 3.50$.

For its short length this book contains a large amount of material. This is made possible in part by the choice of subject matter, but more importantly, by the beautifully compact proofs which the author has been able to construct in many instances. One may assume that the choice of material has been motivated to some extent by the author's preference for those subjects to which she herself has contributed most successfully. The result is a book largely complementary to that of Boas, which deals primarily with functions of exponential type [Entire functions, New York, 1954; this Bulletin 62 (1956) 57-62].

The main theme of the present book is the study of analytic functions which are of finite order in an angle. Whereas the results in Boas's book on this point may be interpreted as results on functions of mean type, the present author achieves complete generality by the use of proximate orders. The second important topic not covered by Boas is the distribution of the values of an analytic function, notably the theories of exceptional values and of lines of Julia.

Like Boas's, the present book should be well within the reach of any student who has taken a standard year course in complex analysis.

A description of the chapters in the book follows.

I. Preliminary results. II. Integral functions of finite order: Hadamard's factorization theorem, Borel and Picard exceptional values, asymptotic values. III. The Phragmén-Lindelöf principle. Various formulations are given of the principle that a not-too-large analytic function in a half-plane which is bounded by $M$ on the frontier is bounded by $M$ throughout. The indicator function 